\section{Hepatic Portal Venous Gas and Severe Right Shoulder Pain after Caesarean Section: A Case Report}

\begin{abstract}
Keywords: Caesarean section; Portal venous gas; Shoulder pain; Computerize tomography

Abstract

A radiological image compatible with the presence of hepatic portal venous gas may associate with severe life threatening diseases requiring urgent operative interventions. Although shoulder pain may be experienced after caesa rean sections, its intensity is usually mild. In this paper, we aimed to present a woman experienced a very severe shoulder pain after a caesarean section and finally diagnosed with the presence of hepatic portal venous gas. To our best knowledge, hepatic portal venous gas presentation associated with caesarean section has not been reported previously. The clinical and radiologica findings of the case were discussed in the light of the medical literature.
\end{abstract}

\section{Introduction}

A radiological finding suggesting the presence of gas in the hepatic portal vein (HPV) is rarely encountered and the pathological process leading to entity is not clearly defined.

Since its first description by Wolfe and Evans in 1955 with a plain radiography, the presence of gas in HPV has been associated with numerous diseases of varying clinical importance. Those included a variety of conditions including some mild disease states originating from extra abdominal causes. However, in some instances the associated conditions required urgent surgical intervention [1].

Although, post operative shoulder pain is not uncommon after laparoscopic surgeries, it is rarely seen after open surgeries, such as caesarean sections [2]. In addition, the intensity of the shoulder pain following caesarean section, if ever exists, is usually mild.

In this paper, we aimed to present a woman experienced a very severe shoulder pain after a caesarean section and finally diagnosed with the presence of hepatic portal venous gas. The clinical and radiological findings of the case were discussed in the light of the medical literature.

\section{Case Report}

A 30-year old, gravidity 3, parity 2 woman was admitted to the obstetrics department of our hospital with the symptoms of the onset of labor. According to the date of last menstrual period and ultrasound confirmation, she had a pregnancy compatible with 39 weeks and 4 days. Her medical and obstetric histories were unremarkable.

Vaginal examination revealed a cervical dilatation and effacement of $2 \mathrm{~cm}$ and $\% 60$, respectively, along with the accumulation of xanthochromic amniotic fluid in the posterior vaginal fornix. Although the fetal heart rate was auscultated within normal limits,

\section{Journal of Andrology \& Gynaecology}

\author{
Ahmet Said Cil ${ }^{1}$, Murat Bozkurtt ${ }^{2 *}$, H. Duygu Kara \\ Bozkurt $^{3}$, Kahraman Ulker ${ }^{2}$ \\ ${ }^{1}$ Department of Radiology, Universal Hospitals Group, Malatya, \\ TURKEY \\ ${ }^{2}$ Department of Obstetrics and Gynecology, Kafkas University \\ School of Medicine, Kars, TURKEY \\ ${ }^{3}$ Department of Radiology, Kafkas University School of Medicine, \\ Kars, TURKEY
}

\section{*Address for Correspondence}

Murat Bozkurt, MD, Assistant Professor, Kafkas University School of Medicine, Department of Obstetrics and Gynecology, KARS, TURKEY; Tel: +905322279072 +905056330044; Fax: 047422514 30; E-mail: jindrmb@yahoo.com

Submission: 18 May 2014

Accepted: 13 June 2014

Published: 16 June 2014

Reviewed \& Approved by: Dr. Robert Shapiro, Assistant Clinical

Professor of Obstetrics and Gynecology at West Virginia University Hospital, USA

non-stress test revealed a non-reactive fetal heart rate pattern associated with late decelerations.

Depending on the diagnosis of fetal distress, the woman was prepared for a caesarean section as usual and a $3500 \mathrm{~g}, 55 \mathrm{~cm}$ boy was delivered with an uneventful cesarean section. The first minute Apgar score of the neonate was 8 .

On post operative day 1, the woman complained of a severe, intolerable right shoulder pain spreading to the right side of the chest and accompanied with a difficulty in respiration.

Depending on the suspicion of postoperative pulmonary embolism, we performed a chest X-ray examination which revealed the accumulation of free gas under the right side of the diaphragm (Figure 1). The demonstration of the levels of serum D-dimer and

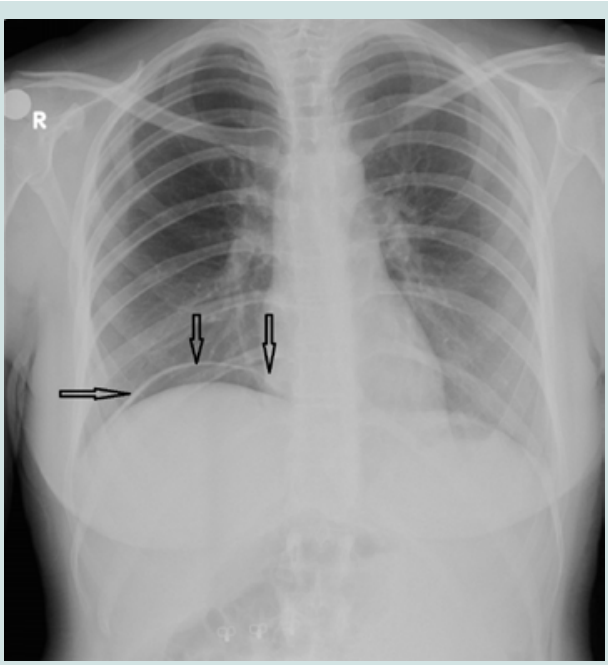

Figure 1: Free air under the right side of diaphragm was seen on AP chest $\mathrm{X}$ - ray graph (open arrows). Note that there was not seen any gas in the liver. 
Citation: Cil AS, Bozkurt M, Kara Bozkurt HD, Ulker K. Hepatic Portal Venous Gas and Severe Right Shoulder Pain after Caesarean Section: A Case Report. J Androl Gynaecol. 2014;2(1): 3.

arterial blood gas saturations within the normal reference ranges weakened the probability of pulmonary embolism. Thus, we performed an abdominal ultrasound examination to identify the probable pathologies that might be related with the abdominal free air. However, the ultrasound findings were unremarkable. Therefore, we needed to perform an abdominal computerized tomography (CT) examination.

CT examination revealed pneumoperitoneum and partial subcutaneous emphysema. In addition, there was a small amount of air inside the left liver lobe suggesting the presence of gas in HPV (Figure $2 \mathrm{~A}, \mathrm{~B}$ ).

After appropriate analgesic management and the mobilization of the woman, the symptoms resolved. Finally, the woman was discharged uneventfully on postoperative day 2 .

\section{Discussion}

We demonstrated free air in sub-diaphragmatic space and HPV in a woman with severe right shoulder and chest pain after a caesarean section. Gas presence in HPV may contribute in the severity of the experienced symptoms. To our knowledge hepatic portal venous gas presentation has not been reported previously after a caesarean section.

Following laparotomies including caesarean section, a small amount of free air may accumulate in the intra-peritoneal space. The condition is referred to as "pneumoperitonum" and is usually asymptomatic [2].

With the popularization of the laparoscopic surgeries, in which $\mathrm{CO} 2$ gas is used to inflate the abdominal wall in order to provide a surgical space, the number of publications referring to iatrogenic pneumoperitonum and its side effects increased $[3,4]$. Shoulder pain and associated dyspnea are the most widely known and demonstrated symptoms experienced following the accumulation of gas in the abdominal viscera [4]. It is believed that the accumulated gas under the diaphragm disrupts the diaphragmatic mobility and irritates the phrenic nerve which in turn, causes the stimulation of the fourth cervical nerve. Thus, the perceived location of the pain is projected to the shoulder area [5].
The existing medical literature lacks the data defining the pathophysiology of shoulder pain experienced after caesarian sections. Radiological demonstration of free gas under the diaphragm and in HPV in our case may provide evidence to explain the rationale of the right shoulder pain and respiratory difficulty.

The disorders associated with radiological images of gas presence in HPV were classified into two main groups as belonging to abdominal and extra abdominal causes. Abdominal disorders included the intestinal wall alterations (e.g. mesenteric ischemia), gastric and bowel dilatation, and intra abdominal infections (e.g. diverticulitis, pylephlebitis). Extra abdominal disorders included the use of corticosteroid therapy and cardio pulmonary resuscitation [6,7].

Most probable explanation of the mechanism of the presence of gas in HPV is based on two theories. The first theory is related with mechanic actions. It suggests that traumatic manipulation of the intestines leads to gas passage into the venous system [6] and the second theory suggests the passage of gas produced by microorganisms on intestinal wall, into mesenteric veins and then to the portal vein [68]. However, our surgical procedure was uneventful and we did not have to manipulate the intestines extensively. Truly we did not touch the intestines. In addition, the woman neither developed pyrexia nor any other sign of an infection. Thus, the aforementioned theories seem to insufficient to explain the presence of gas in HPV in our case. However, as a matter of fact, the presence of gas in porto-mesenteric veins is assumed as idiopathic in approximately $15 \%$ of cases $[7,9]$.

During laparoscopic surgeries, $\mathrm{CO}_{2}$ passing into the circulation from intra abdominal visceral surfaces and peritoneum may cause hypercapnia and acidosis. Absorbed $\mathrm{CO}_{2}$ is exhaled from the lungs [3]. However, to the best of our knowledge, there is no publication on accumulation of gas in portal veins during or after the laparoscopic surgeries. The studies about the composition of HPV gas clearly suggest that it includes large amounts of $\mathrm{CO}_{2}[3,4]$. However, in our case we did not know the composition of the accumulated gas. Unlike the laparoscopic surgery, the intra-abdominal gas is not composed of $\mathrm{CO}_{2}$, but different gases like nitrogen and oxygen.

Gas in HPV can be demonstrated by direct radiography, US
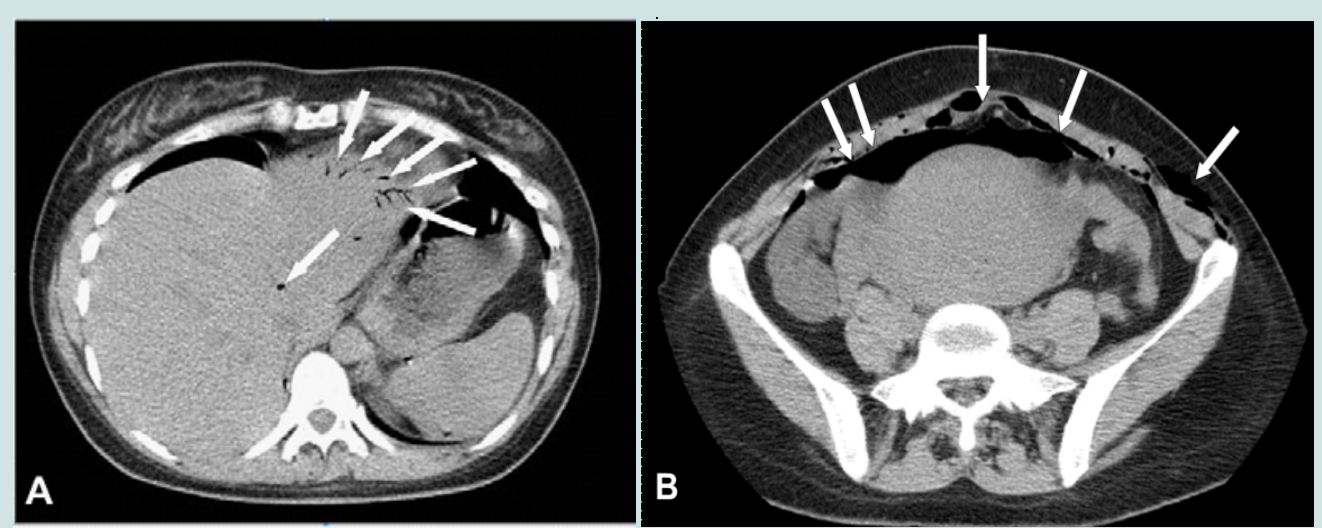

Figure 2: Computerized tomography images show A) air in the liver parenchyma located at the periphery of the left liver lobe matching with peripheral branch of left portal vein (arrows). B) Uterine involution has not been completed yet after the pregnancy. Free air in the abdominal viscera and minimal subcutaneous emphysema on left side are seen (white arrows). 
Citation: Cil AS, Bozkurt M, Kara Bozkurt HD, Ulker K. Hepatic Portal Venous Gas and Severe Right Shoulder Pain after Caesarean Section: A Case Report. J Androl Gynaecol. 2014;2(1): 3.

and CT. However, small amounts of gas may not be seen in US and radiographies. In this manner, positive and negative predictive value of CT is higher than other imaging modalities [7,9]. In a typical CT examination, portal vein gas appears as tubular areas of decreased attenuation in the liver, predominantly in periphery of the left lobe by centrifugal blood flow [7].

Intra hepatic portal vein gas may be confused with pneumobilia (air in the biliary tree). In pneumobilia, the air is located in the central sections of liver and accompanied with the air images in extra hepatic great bile ducts, such as common bile duct and common hepatic duct. In comparison with the right hepatic lobe left hepatic lobe is more ventrally located which causes the predilection of pneumobilia to the left lobe. In contrast, images of portal vein gas accumulation are obtained at the peripheral tissues beneath the hepatic capsule and constitute smaller, numerous areas. Although they are different entities, pneumobilia and portal vein gas may coexist [7]. We could not demonstrate gas on X-ray and ultrasound examinations, but on CT examination, during our patient's work up which might result from relatively lower gas amount.

PVG and sub diaphragmatic free air are radiologic signs indicating severe intra abdominal pathologies, particularly in case where they are demonstrated in plain radiographic images. In conditions where CT demonstrates the actual primary disease, these findings are usually underestimated. However, presence of the findings mandates a comprehensive examination for a differential diagnosis work [9-12]

In conclusion, intra-abdominal accumulation of air after laparotomies, such as a caesarian section, may cause severe shoulder pain associated with and small amount of gas in the portal venous system. CT seems to be the most affective imaging modality for differential diagnosis. After ruling out major pathological conditions, simple analgesic use is adequate to treat shoulder pain and treatment

\section{for PVG is not necessary.}

\section{References}

1. Wolf JN, Evans WA (1955) Gas in the portal veins of the liver in infants: a roentgenographic demonstration with postmortem anatomical correlation. Am J Roentgenol Radium Ther Nucl Med 74: 486- 468.

2. Zirak N, Soltani G, Hafizi L, Mashayekhi Z, Kashani I (2012) Shoulder pain after caesarean section: comparison between general and spinal anaesthesia. J Obstet Gynaecol 32: 347- 379.

3. Jackson SA, Laurence AS, Hill JC (1996) Does post-laparoscopy pain relate to residual carbon dioxide? Anaesthesia 51: 485-487.

4. Alexander JI (1997) Pain after laparoscopy. Br J Anaesth; 79: 369-378.

5. Mouton WG, Bessell JR, Otten KT, Maddern GJ (1999) Pain after laparoscopy. Surg Endosc 13: 445-448.

6. Liebman PR, Patten MT, Manny J, Benfield JR, Hechtman HB (1978) Hepatic-portal venous gas in adults: Etiology, pathophysiology and clinical significance. Ann Surg 187: 281-287.

7. Sebastia' C, Quiroga S, Espin E, Boye' R, Alvarez-Castells A, et al. (2000) Portomesenteric vein gas: Pathologic mechanisms, CT findings and prognosis. Radiographics 20: 1213- 1224.

8. Hong JJ, Gadaleta D, Rossi P, Esquivel J, Davis JM (1997) Portal vein gas, a changing clinical entity. Report of 7 patients and review of the literature. Arch Surg 132: 1071-1075.

9. Scheidler J, Stäbler A, Kleber G, Neidhardt D (1995) Computed tomography in pneumatosis intestinalis: differential diagnosis and therapeutic consequences. Abdom Imaging 20: 523-528.

10. Mohammed AH, Khot UP, Thomas D (2007) Portal venous gas - case report and review of the literature. Anaesthesia; 62: 400-404.

11. Celoria G, Coe NP (1990) Does the presence of hepatic portal venous gas mandate an operation? A reassessment. South Med J 83: 592- 594.

12. Griffiths DM, Gough MH (1986) Gas in the hepatic portal veins. Br J Sur 73: 172-176. 